\title{
Orientational Phase Transitions in the Hexagonal Phase of a Diblock Copolymer Melt Under Shear Flow
}

\author{
A.N. Morozov, A.V. Zvelindovsky, J.G.E.M. Fraaije \\ Faculty of Mathematics and Natural Sciences, University of Groningen, Nijenborgh 4, 9747 AG \\ Groningen, The Netherlands
}

\begin{abstract}
We generalize the earlier theory by Fredrickson [J. Rheol. 38, 1045 (1994)] to study the orientational behaviour of the hexagonal phase of diblock copolymer melt subjected to steady shear flow. We use symmetry arguments to show that the orientational ordering in the hexagonal phase is a much weaker effect than in the lamellae. We predict the parallel orientation to be stable at low and the perpendicular orientation at high shear rates. Our analysis reproduces the experimental results by Tepe et al. [Macromolecules $\mathbf{2 8}$, 3008 (1995)] and explains the difficulties in experimental observation of the different orientations in the hexagonal phase.
\end{abstract}

\section{INTRODUCTION}

Polymeric liquids subjected to shear flow demonstrate a very peculiar phase behaviour. Their phase diagrams not only contain regions of stability of the different symmetry types (lamellae, hexagonally packed cylinders and so on), but also these regions have an internal structure. Application of shear breaks the rotational symmetry selecting the preferable direction. Thus, at given external parameters (temperature and shear rate) a certain orientation of the symmetry pattern with respect to the selected direction is more stable than the others [四]. 
Experimentally this phenomena was first observed for the lamellar phase [2]. In this work a microphase separated poly(ethylene-propylene)-poly(ethylethylene) diblock copolymer melt was subjected to an oscillatory shear. Near the order-disorder transition (ODT) the lamellae have their normal parallel to the velocity gradient (the parallel orientation) at low shear frequencies, while at high shear frequencies the lamellae have their normal parallel to the vorticity direction of the shear flow (the perpendicular orientation). At lower temperatures the parallel orientation is always the most stable one.

The first theoretical attempt to study the orientational phase transition was by Cates and Milner [3]. They considered the equation of motion for the order parameter with a coupling of applied steady shear to the composition fluctuations. They found that flow completely changes the fluctuation spectrum. As a result, the fluctuations of the order parameter are suppressed and the ODT temperature is raised. The authors suggested that the perpendicular orientation is stable near the ODT since the composition fluctuations with wave vectors normal to both the velocity and the velocity gradient are the least affected by shear.

Later Fredrickson has shown that the angle-dependence of the fourth order vertex function is crucial for construction of a realistic non-equilibrium phase diagram. Within this framework Fredrickson reproduced the experimental observation by Koppi et al. [2]. The theory fails to describe the selection of the parallel orientation at higher frequencies 4 .

In the present paper we generalize the Fredrickson theory to describe the orientational phase transitions in the hexagonal phase of a diblock copolymer melt subjected to steady simple shear flow. The symmetry pattern of this phase consists of hexagonally packed cylinders made up of blocks of one type immersed in the surroundings of the other blocks (see Fig.(1). In equilibrium this phase appears in between lamellae and body-centered cubic phase [5]:6]. The experiments under shear flow show an analogy with the orientational behaviour of the lamellar phase $[7$ 9]. In slow flow the symmetry pattern has "2 dots up", while in the faster flow the "1 dot up" orientation appears (see Fig.21). In order to keep universality we call them the parallel and the perpendicular orientations, respectively. There have been many 
observations of the ordering of the hexagonal phase subjected to shear flow [7 9]. Recently, Tepe et al. have varied temperature and shear rate and observed both orientations of the hexagonal pattern in a non-symmetric polyethylene-poly(ethylenepropylene) (PE-PEP) diblock copolymer melt. We schematically present their results in Fig.2. Our goal is to reproduce this dynamical phase diagram theoretically.

We start with a speculative analogy with lamellae. Both orientations of the hexagonal pattern could be considered as parallel lamellae with different interlamellar distances (see Fig.3). High shear squeezes the pattern and the lamellae with the smallest interlayer distance are favorable. In very slow shear the intermolecular forces resulting in microphase separation will play the predominant role and lamellae with the biggest interlayer distance are stable. Such simple reasoning already reproduces the main features of Fig.2.

We proceed with more exact and motivated analysis. In Section II we formulate a dynamical model and study its equilibrium limit. In Sections III and IV we apply the methods of Fredrickson [1] to analyze the high-shear and low-shear behaviour. In the Conclusions we compare the orientational behaviour of the hexagonal and lamellar phases and explain the origin of difficulties in experimental study of this behaviour.

\section{DYNAMIC EQUATIONS}

Let us consider a diblock copolymer melt. The starting point of our analysis is the dynamic equation for an order parameter $\psi(\mathbf{r})$, which we choose to be a deviation of a local density of monomers of one block from its average value [5]. In the present work we use the Fokker-Planck equation for an incompressible block copolymer melt [1, 3 , 10]:

$$
\frac{\partial P}{\partial t}[\psi, t]=\int_{p} \frac{\delta}{\delta \psi(\mathbf{p})}\left[\mu\left(\frac{\delta}{\delta \psi(-\mathbf{p})}+\frac{\delta H}{\delta \psi(-\mathbf{p})}\right)-D p_{x} \frac{\partial}{\partial p_{y}} \psi(\mathbf{p})\right] P[\psi, t]
$$

where $H[\psi]$ is the Landau-Ginzburg Hamiltonian

$$
H[\psi]=\frac{1}{2} \int_{q}\left[\tau+\left(q-q_{0}\right)^{2}\right] \psi(\mathbf{q}) \psi(-\mathbf{q})+
$$




$$
\begin{aligned}
& \frac{1}{3 !} \int_{q_{1}} \int_{q_{2}} \int_{q_{3}} \xi\left(\mathbf{q}_{1}, \mathbf{q}_{2}, \mathbf{q}_{3}\right) \psi\left(\mathbf{q}_{1}\right) \psi\left(\mathbf{q}_{2}\right) \psi\left(\mathbf{q}_{3}\right)+ \\
& \frac{1}{4 !} \int_{q_{1}} \int_{q_{2}} \int_{q_{3}} \int_{q_{4}} \lambda\left(\mathbf{q}_{1}, \mathbf{q}_{2}, \mathbf{q}_{3}, \mathbf{q}_{4}\right) \psi\left(\mathbf{q}_{1}\right) \psi\left(\mathbf{q}_{2}\right) \psi\left(\mathbf{q}_{3}\right) \psi\left(\mathbf{q}_{4}\right)
\end{aligned}
$$

and $\mu$ is the Onsager mobility coefficient, which we assume to be constant (see [1, 11, 12 for discussion).

For a certain configuration of the field $\psi(\mathbf{q})$, the function $P[\psi]$ gives the probability of its realizations. In the stationary state, $P[\psi]$ reduces to the Boltzmann distribution $P \sim \exp (-H[\psi])$ in the limit $D=0$ [13].

In the derivation of the equation (2.1) we assumed the standard flow geometry $\mathbf{v}=D y \mathbf{e}_{x}$. It means that we ignored any alteration of the velocity profile caused by the internal structure of the melt. Fredrickson has showed [1] that by taking into account different viscosities of the two blocks one can approximate the real velocity profile by the same functional form $\mathbf{v}=D_{\text {eff }} y \mathbf{e}_{x}$. Here $D_{\text {eff }}$ is a renormalized shear rate:

$$
D_{\mathrm{eff}}=D(1+\text { corrections })
$$

In lamellar phase at high shear, the lamellae are perpendicular at high temperatures, and parallel at low temperatures. Fredrickson has shown that the orientation-dependent hydrodynamic corrections are needed to explain this transition. In hexagonal phase such an effect at high shear is not known, and we postpone hydrodynamic corrections to future analysis. Here we approximate $D_{\text {eff }}$ by $D$.

We take the first and the second cumulants to obtain the equations for

$$
\begin{aligned}
& C(\mathbf{p})=\langle\psi(\mathbf{p})\rangle \\
& S(\mathbf{p})=\langle\psi(\mathbf{p}) \psi(-\mathbf{p})\rangle-\langle\psi(\mathbf{p})\rangle\langle\psi(-\mathbf{p})\rangle
\end{aligned}
$$

The resulting equations can be greatly simplified if we use the principle-harmonic approximation for the mean density profile:

$$
C(\mathbf{p})=\sum_{i=1}^{n} a_{i}\left[\delta_{\mathbf{p}, q_{0} \mathbf{n}^{(i)}}+\delta_{\mathbf{p},-q_{0} \mathbf{n}^{(i)}}\right]
$$


where the set of vectors $\mathbf{n}^{(i)}$ determine the lattice symmetry. The equation (2.5) says that all structures in the system have the same typical size $\sim 1 / q_{0}$, where $q_{0}$ corresponds to the primary peak of the structure factor $S(\mathbf{p})$ (see the first term in (2.2)).

For the steady state the equations transform to:

$$
\begin{aligned}
h_{i}= & \tau a_{i}+\mathfrak{A}_{i}+\frac{1}{2} a_{i} \int_{q} \lambda\left(q_{0} \mathbf{n}^{(i)},-q_{0} \mathbf{n}^{(i)}, \mathbf{q},-\mathbf{q}\right) S(\mathbf{q})+\mathfrak{B}_{i} \\
1= & -\frac{D}{2 \mu} p_{x} \frac{\partial}{\partial p_{y}} S(\mathbf{p})+ \\
& S(\mathbf{p})\left[\tau+\left(p-q_{0}\right)^{2}+\frac{1}{2} \int_{q} \lambda(\mathbf{p}, \mathbf{q},-\mathbf{p},-\mathbf{q}) S(\mathbf{q})+\mathfrak{C}\right]
\end{aligned}
$$

where we defined the structure constants:

$$
\begin{aligned}
\mathfrak{A}_{i} & =\frac{1}{2} \int_{q_{1}} \int_{q_{2}} \xi\left(-q_{0} \mathbf{n}^{(i)}, \mathbf{q}_{1}, \mathbf{q}_{2}\right) C\left(\mathbf{q}_{1}\right) C\left(\mathbf{q}_{2}\right) \\
\mathfrak{B}_{i} & =\frac{1}{3 !} \int_{q_{1}} \int_{q_{2}} \int_{q_{3}} \lambda\left(-q_{0} \mathbf{n}^{(i)}, \mathbf{q}_{1}, \mathbf{q}_{2}, \mathbf{q}_{3}\right) C\left(\mathbf{q}_{1}\right) C\left(\mathbf{q}_{2}\right) C\left(\mathbf{q}_{3}\right) \\
\mathfrak{C} & =\frac{1}{2} \int_{q} \lambda(\mathbf{p}, \mathbf{q},-\mathbf{p},-\mathbf{q}) C(\mathbf{q}) C(-\mathbf{q})
\end{aligned}
$$

In equation (2.6) we introduced artificial external fields $h_{i}$. This is equivalent to an introduction of the additional term in the Hamiltonian $H_{e x t}=-\int_{q} h(\mathbf{q}) \psi(-\mathbf{q}), h(\mathbf{q})=$ $\sum_{i=1}^{n} h_{i}\left[\delta_{\mathbf{q}, q_{0} \mathbf{n}^{(i)}}+\delta_{\mathbf{q},-q_{0} \mathbf{n}^{(i)}}\right]$, which describes the interaction with an external field $h(\mathbf{q})$. The fields $h_{i}$ will allow us to construct a potential $\Phi$ governing the dynamics. In equilibrium the potential $\Phi$ has the meaning of the free energy of the system. Thus, by introducing the fields $h_{i}$ we obtain an analytic continuation of the free energy to the dynamic case [1]. This is possible because our model is conservative [14].

As our last simplification we introduce an approximation for the vertex functions $\xi$ and $\lambda$. Since we use the principle-harmonic approximation in (2.5), we assume that all wavevectors have the same modulus $|\mathbf{q}|=q_{0}$. Moreover, following [15] 17], we take into account the weak angle-dependence in the 4th-order vertex function $\lambda$, so

$$
\begin{aligned}
\xi\left(\mathbf{q}_{1}, \mathbf{q}_{2}, \mathbf{q}_{3}\right) & =\xi \delta\left(\hat{\mathbf{q}}_{1}+\hat{\mathbf{q}}_{2}+\hat{\mathbf{q}}_{3}\right) \\
\lambda\left(\mathbf{q}_{1}, \mathbf{q}_{2},-\mathbf{q}_{1},-\mathbf{q}_{2}\right) & =\lambda\left[1-\beta\left(\hat{\mathbf{q}}_{1} \cdot \hat{\mathbf{q}}_{2}\right)^{2}\right] \\
\beta & \ll 1
\end{aligned}
$$


where $\hat{\mathbf{q}}=\mathbf{q} / q$ denotes the unit vector in the direction of $\mathbf{q}$.

To make the general equations (2.6) and (2.7) specific for the hexagonal phase one needs to calculate the structure constants (2.8) taking into account the symmetry of the phase. The average density profile for the hexagonally packed cylinders is given by (2.5), where the basis vectors $\mathbf{n}^{(i)}$ are:

$$
\begin{aligned}
& \mathbf{n}^{(1)}=\{0, \cos \phi, \sin \phi\} \\
& \mathbf{n}^{(2)}=\frac{1}{2}\{0,-\cos \phi-\sqrt{3} \sin \phi, \sqrt{3} \cos \phi-\sin \phi\} \\
& \mathbf{n}^{(3)}=\frac{1}{2}\{0,-\cos \phi+\sqrt{3} \sin \phi,-\sqrt{3} \cos \phi-\sin \phi\}
\end{aligned}
$$

The angle $\phi$ defines the orientation of the hexagonal pattern. The case $\phi=0$ corresponds to the parallel orientation in real space (see Fig.2).

Using (2.8), 2.11) and (2.9), (2.10) we obtain for the structure constants:

$$
\begin{aligned}
\mathfrak{A}_{i} & =\xi a_{k} a_{l} \\
\mathfrak{B}_{i} & =a_{i} \lambda\left[a_{k}^{2}\left(1-\frac{\beta}{4}\right)+a_{l}^{2}\left(1-\frac{\beta}{4}\right)+\frac{1}{2} a_{i}^{2}(1-\beta)\right] \\
\mathfrak{C} & =\lambda\left[a_{1}^{2}\left(1-\beta\left(\hat{\mathbf{p}} \cdot \mathbf{n}^{(1)}\right)^{2}\right)+a_{2}^{2}\left(1-\beta\left(\hat{\mathbf{p}} \cdot \mathbf{n}^{(2)}\right)^{2}\right)+a_{3}^{2}\left(1-\beta\left(\hat{\mathbf{p}} \cdot \mathbf{n}^{(3)}\right)^{2}\right)\right] \\
& i \neq k \neq l
\end{aligned}
$$

We introduce the notation:

$$
\begin{aligned}
\sigma(\hat{\mathbf{p}}) & =\frac{\lambda}{2} \int_{q} S(\mathbf{q})\left[1-\beta(\hat{\mathbf{p}} \cdot \hat{\mathbf{q}})^{2}\right] \\
r-\hat{\mathbf{p}} \cdot \overleftrightarrow{\mathbf{e}} \cdot \hat{\mathbf{p}} & =\tau+\sigma(\hat{\mathbf{p}})+\lambda \sum_{i=1}^{3} a_{i}^{2}\left[1-\beta\left(\hat{\mathbf{p}} \cdot \mathbf{n}^{(i)}\right)^{2}\right] \\
S_{0}(\mathbf{p}) & =\left[r-\hat{\mathbf{p}} \cdot \stackrel{\leftrightarrow}{\mathbf{e}} \cdot \hat{\mathbf{p}}+\left(p-q_{0}\right)^{2}\right]^{-1}
\end{aligned}
$$

and rewrite the steady-state equations in the final form:

$$
\begin{gathered}
-\frac{D}{2 \mu} p_{x} \frac{\partial}{\partial p_{y}} S(\mathbf{p})+S(\mathbf{p}) S_{0}^{-1}(\mathbf{p})=1 \\
h_{i}=\left(r-\hat{\mathbf{n}}^{(i)} \cdot \stackrel{\leftrightarrow}{\mathbf{e}} \cdot \hat{\mathbf{n}}^{(i)}\right) a_{i}+\xi a_{k} a_{l}-\frac{1}{2} \lambda a_{i}^{3}(1-\beta) \\
i \neq k \neq l
\end{gathered}
$$


Notation (2.13)-(2.15) has a clear physical meaning. The fluctuation integral (2.13) takes into account the fluctuations of the order parameter 18 and renormalizes the temperature in the system. Because of the angle-dependence of the 4th-order vertex function $\lambda$ (eq.2.10), the renormalized temperature also has an angle-dependence. Expanding it to the first order in $\beta$ (one can easily check that the anisotropy tensor $e_{i j}$ is of order $O(\beta)$ ), we extract this angle-dependence and get (2.14), where $r$ denotes the $\mathbf{p}$-independent part of the renormalized

temperature and $-\hat{\mathbf{p}} \cdot \stackrel{\leftrightarrow}{\mathbf{e}} \cdot \hat{\mathbf{p}}$ adsorbs the other terms. Finally, $S_{0}(\mathbf{p})$ is an equilibrium structure factor, which in the limit $\beta=0$ reduces to the one studied by Brazovskii [18], Fredrickson and Helfand [6].

\section{Equilibrium analysis}

The equations (2.16) and (2.17) describe the behaviour of the hexagonal phase at any shear rate. For the particular case $(D=0, \beta=0)$ these equations were studied in a number of articles [6, [12, [8]. Before studying the dynamics we want to show the influence of the angle-dependence in $\lambda$ (2.10) on the equilibrium phase behaviour, so we construct the free energy at equilibrium $D=0$. The equation (2.16) reads:

$$
S(\mathbf{p})=S_{0}(\mathbf{p})=\left[r-\hat{\mathbf{p}} \cdot \stackrel{\leftrightarrow}{\mathbf{e}} \cdot \hat{\mathbf{p}}+\left(p-q_{0}\right)^{2}\right]^{-1}
$$

Performing the integration in (2.13) up to the first order in $\beta$ we get:

$$
\sigma(\hat{\mathbf{p}}) \equiv \sigma_{e q}=\frac{\alpha \lambda}{\sqrt{r}}\left[1-\frac{1}{3} \beta(\hat{\mathbf{p}} \cdot \hat{\mathbf{p}})+\frac{e_{i i}}{6 r}\right]
$$

Here $e_{i i}$ denotes the trace of the anisotropy tensor $\stackrel{\leftrightarrow}{\mathbf{e}}$. Separating $\hat{\mathbf{p}}$-dependent terms in $\sigma(\hat{\mathbf{p}})$ with the help of (2.14) we obtain:

$$
\begin{aligned}
r & =\tau+\frac{\alpha \lambda}{\sqrt{r}}+\frac{\alpha \lambda}{6 r^{3 / 2}} e_{i i}+\lambda\left(a_{1}^{2}+a_{2}^{2}+a_{3}^{2}\right) \\
e_{i j} & =\frac{\alpha \lambda}{\sqrt{r}} \frac{\beta}{3} \delta_{i j}+\beta \lambda\left[a_{1}^{2} n_{i}^{(1)} n_{j}^{(1)}+a_{2}^{2} n_{i}^{(2)} n_{j}^{(2)}+a_{3}^{2} n_{i}^{(3)} n_{j}^{(3)}\right]
\end{aligned}
$$

The equations of motion for $a_{i}$ (2.17) have a potential form: 


$$
h_{i}=\frac{1}{2} \frac{\partial \Phi}{\partial a_{i}}
$$

To integrate $(2.22)$ one needs to take into account dependence of $r$ on $a_{i}$ and treat an integral of a composite function:

$$
\int_{0}^{a_{i}} d a_{i}(\ldots)=\int_{r 0}^{r} d r \frac{\partial a_{i}}{\partial r}(\ldots)
$$

where the Jacobian $\frac{\partial a_{i}}{\partial r}$ can be found from (2.20), and $r_{0}$ is a temperature at which the disordered phase loses stability, given by equation (2.20) with $a_{1}=a_{2}=a_{3}=0$. Integrating (2.22) we obtain the free energy:

$$
\begin{aligned}
& \Phi=\Phi^{\prime}+\Phi_{\text {str }} \\
& \Phi^{\prime}=\frac{r^{2}-r_{0}^{2}}{2 \lambda}+\alpha\left(1-\frac{\beta}{2}\right)\left(\sqrt{r}-\sqrt{r_{0}}\right)+\frac{1}{2} \alpha \tau \beta\left(\frac{1}{\sqrt{r}}-\frac{1}{\sqrt{r_{0}}}\right)+\frac{1}{6} \alpha^{2} \lambda \beta\left(\frac{1}{r}-\frac{1}{r_{0}}\right) \\
& \Phi_{\text {str }}=-\frac{1}{4} \lambda(1+\beta)\left(a_{1}^{4}+a_{2}^{4}+a_{3}^{4}\right)+2 \xi a_{1} a_{2} a_{3}-\frac{1}{4} \beta \lambda\left(a_{1}^{2} a_{2}^{2}+a_{1}^{2} a_{3}^{2}+a_{2}^{2} a_{3}^{2}\right)
\end{aligned}
$$

where we have separated terms determined by the structure constants.

The amplitudes $a_{i}$ that minimize the potential $\Phi$ are found by solving the set of equations $h_{i}=0$. Its most important feature is that $\sigma$ does not depend on the $\mathbf{n}^{(i)}$. Thus, the resulting equations are isotropic with respect to $a_{i}$ and the solution has the form $a_{1}=a_{2}=a_{3}=\mathfrak{a}$.

To explore the stability region of the hexagonal phase we need to solve the resulting system of equations for given temperature and composition:

$$
\begin{aligned}
& r=\tau+\frac{\alpha \lambda}{\sqrt{r}}+\frac{(\alpha \lambda)^{2}}{6 r^{2}} \beta+\frac{\alpha \lambda^{2}}{2 r^{3 / 2}} \mathfrak{a}^{2} \beta+3 \lambda \mathfrak{a}^{2} \\
& r_{0}=\tau+\frac{\alpha \lambda}{\sqrt{r_{0}}}+\frac{(\alpha \lambda)^{2}}{6 r_{0}^{2}} \beta \\
& r-\frac{1}{3} \beta \frac{\alpha \lambda}{\sqrt{r}}-\lambda \mathfrak{a}^{2}\left(\frac{1}{2}+\beta\right)+\xi \mathfrak{a}=0 \\
& \Phi=\Phi^{\prime}-\frac{3}{4} \lambda \mathfrak{a}^{4}(1+2 \beta)+2 \xi \mathfrak{a}^{3}
\end{aligned}
$$

The calculation technique of Fredrickson and Helfand [6] allows us to demonstrate the influence of the $\beta$-terms on the equilibrium phase diagram. In Fig. 1 and Fig.5 we show that the approximation (2.10) results in a non-significant quantitative shift of values. The effect 
of $\beta$ on the equilibrium phase diagram is tiny, but in the next section we will demonstrate that $\beta$ is dominant in explaining the dynamical orientational ordering.

\section{STRONG-SHEAR BEHAVIOUR}

To study the orientational dynamics in the strong-shear regime we need to solve (2.16) and (2.17) in the limit $D \rightarrow \infty$. In this case it is impossible to obtain a solution of (2.16) as a perturbation series in $1 / D$. Instead, we use approach developed by Cates and Milner [3]. One can apply the RG methods [10] to find the asymptotic behaviour of the structure factor $S(\mathbf{p}, D \rightarrow \infty)=S_{\infty}(\mathbf{p})$. Then, at large shear rates the structure factor can be

approximated by interpolating between $S_{0}(\mathbf{p})$ and $S_{\infty}(\mathbf{p})$. Cates and Milner introduced the following approximation:

$$
S^{-1}(\mathbf{p})=S_{0}^{-1}(\mathbf{p})+S_{\infty}^{-1}(\mathbf{p})=r-\mathbf{p} \cdot \stackrel{\leftrightarrow}{\mathbf{e}} \cdot \mathbf{p}+\left(p-q_{0}\right)^{2}+\frac{1}{c_{0}}\left(\frac{D\left|p_{x} p_{y}\right|}{\mu \alpha^{1 / 2}}\right)^{2 / 3}
$$

where $c_{0}=\frac{1}{3}(48 \pi)^{1 / 3} \Gamma\left(\frac{1}{3}\right)$. To the leading order in $D$ the fluctuation integral is equal to [1]:

$$
\sigma(\hat{\mathbf{p}})=(\alpha \lambda)^{2 / 3} \mathfrak{D}\left[I_{1}-\beta\left(I_{2} \hat{p}_{x}^{2}+I_{2} \hat{p}_{y}^{2}+I_{3} \hat{p}_{z}^{2}\right)\right]
$$

where

$$
\mathfrak{D}=\frac{\sqrt{c_{0}}}{(48 \pi)^{1 / 3}}\left(\frac{D^{*}}{D}\right)^{1 / 3}, D^{*}=\mu \lambda \sqrt{\alpha}
$$

and

$$
I_{1}=\frac{\Gamma\left(\frac{1}{2}\right) \Gamma\left(\frac{1}{3}\right)^{2}}{2 \pi \Gamma\left(\frac{7}{6}\right)} \approx 2.2, I_{2}=\frac{\Gamma\left(\frac{1}{2}\right) \Gamma\left(\frac{1}{3}\right) \Gamma\left(\frac{4}{3}\right)}{2 \pi \Gamma\left(\frac{13}{6}\right)} \approx 0.6, I_{3}=\frac{\Gamma\left(\frac{3}{2}\right) \Gamma\left(\frac{1}{3}\right)^{2}}{2 \pi \Gamma\left(\frac{13}{6}\right)} \approx 0.9
$$

For the external fields $h_{i}$ the equation (2.17) results in:

$$
h_{i}=\left[\tau+\sigma\left(\mathbf{n}^{(i)}\right)+\lambda A\left(1-\frac{1}{4} \beta\right)\right] a_{i}+\xi a_{\alpha} a_{\beta}-\frac{1}{2} \lambda a_{i}^{3}\left(1+\frac{1}{2} \beta\right)
$$

where

$$
A=a_{1}^{2}+a_{2}^{2}+a_{3}^{2}
$$


The potential $\Phi$ is given by (2.22). Unlike the equilibrium situation we do not have any problem with integration $\left((\underline{3.3})\right.$ does not depend on both $r$ and $\left.e_{i j}\right)$, and the equation for the free energy is straightforward:

$$
\Phi=\tau A+\sum_{j=1}^{3} \sigma\left(\mathbf{n}^{(j)}\right) a_{j}^{2}+\frac{1}{2} \lambda\left(1-\frac{1}{4} \beta\right) A^{2}+2 \xi a_{1} a_{2} a_{3}-\frac{1}{4} \lambda\left(1+\frac{1}{2} \beta\right) \sum_{j=1}^{3} a_{j}^{4}
$$

The amplitudes $a_{i}$ are the solutions of the equations

$$
h_{i}=0
$$

In the absence of the term $\sigma\left(\mathbf{n}^{(j)}\right) a_{j}^{2}$ these equations have a uniform solution $a_{1}=a_{2}=$ $a_{3}$. The presence of this term breaks the symmetry of the equations and allows for the nonequal amplitudes. We construct the solutions of (3.5) as a perturbation series in two small parameters: $\mathfrak{D}$ and $\beta$. The highest order in the perturbation series for the amplitudes is determined by the following argument. Our goal is to obtain an angle-dependent free energy. This angle-dependence can only appear via various but symmetric combinations of the basis vectors $\mathbf{n}^{(i)}$. Let us discuss an example:

$$
n_{y}^{(1) 2 x}+n_{y}^{(2) 2 x}+n_{y}^{(3) 2 x}
$$

This combination is angle-dependent if $x \geq 3$. One can easily check that it is also true for the other symmetric combinations. The only way for such a combination to enter the equation for the free energy is via a term like $\sigma^{3}$, which is proportional to $\beta^{3} \mathfrak{D}^{3}$. Thus, in what follows we keep terms up to $O\left(\beta^{3} \mathfrak{D}^{3}\right)$. Solving (3.3) for the amplitudes and substituting them into (3.4), we obtain

$$
\Phi=\Phi_{0}+\Phi_{1} \mathfrak{D}+\Phi_{2} \mathfrak{D}^{2}+\Phi_{3} \mathfrak{D}^{3}
$$

The coefficients $\Phi_{i}$ are given by:

$$
\begin{aligned}
& \Phi_{0}=\frac{-2+15(2-5 x) x-2(1-10 x)^{3 / 2}}{125} \frac{\xi^{4}}{\lambda^{3}} \\
& \Phi_{1}=\frac{3}{25} I_{1}(1+\sqrt{1-10 x})^{2} \frac{\xi^{2} \alpha^{2 / 3}}{\lambda^{4 / 3}}
\end{aligned}
$$




$$
\begin{aligned}
& \Phi_{2}=-\frac{3}{5} I_{1}^{2}\left(1+\frac{1}{\sqrt{1-10 x}}\right)\left(\alpha^{4} \lambda\right)^{1 / 3} \\
& \Phi_{3}=\left[-\frac{I_{1}^{3}}{(1-10 x)^{3 / 2}}+\left(I_{3}-I_{2}\right)^{3} \beta^{3} \frac{\sqrt{1-10 x}(122-5 x)+27(14-5 x)}{16(8+x)^{3}} \cos 6 \phi\right]\left(\frac{\alpha \lambda}{\xi}\right)^{2}
\end{aligned}
$$

Here we have introduced the dimensionless temperature $x=\tau \lambda / \xi^{2}$ and kept only the leading terms in $\beta$ for a given order in $\mathfrak{D}$. The second term in $\Phi_{3}$ is the angle-dependent contribution we were looking for.

The spinodal temperature is obtained from the condition: $\left.\Phi\right|_{\tau=\tau_{s}}=0$. It reads:

$$
\tau_{s}=\tau_{s}^{(0)}+\tau_{s}^{(1)} \mathfrak{D}+\tau_{s}^{(2)} \mathfrak{D}^{2}+\tau_{s}^{(3)} \mathfrak{D}^{3}
$$

where:

$$
\begin{aligned}
\tau_{s}^{(0)} & =\frac{4}{9(5-2 \beta)} \frac{\xi^{2}}{\lambda} \\
\tau_{s}^{(1)} & =-\left[I_{1}-\frac{1}{2} \beta\left(I_{2}+I_{3}\right)\right](\alpha \lambda)^{2 / 3} \\
\tau_{s}^{(2)} & =\frac{9 \beta^{2}(5-2 \beta)^{2}\left(I_{3}-I_{2}\right)^{2}}{64(13-7 \beta)} \frac{\alpha^{4 / 3} \lambda^{7 / 3}}{\xi^{2}} \\
\tau_{s}^{(3)} & =-\frac{81\left(I_{3}-I_{2}\right)^{3} \beta^{3}(5-2 \beta)^{5}}{512(13-7 \beta)^{3}}\left(\frac{\alpha \lambda^{2}}{\xi^{2}}\right)^{2} \cos 6 \phi
\end{aligned}
$$

Finally, we calculate the transition temperature between cylinders and lamellae. Up to the first order in $\mathfrak{D}$ we obtain:

$$
\tau_{t r}=-\frac{7+3 \sqrt{6}}{5} \frac{\xi^{2}}{\lambda}+I_{1}(7+3 \sqrt{6})(\alpha \lambda)^{2 / 3} \mathfrak{D}
$$

Now we summarize our results. In the high-shear limit the hexagonal phase is found to be stable in the temperature range from $\tau_{s}$ to $\tau_{t r}$ (see Fig.6). Here we suppose that hexagonal phase is the first phase appearing at cooling down from melt. If this is not a case, the temperature range $\left(\tau_{s}, \tau_{t r}\right)$ transforms to $\left(\tau_{*}, \tau_{t r}\right)$, where $\tau_{*}$ is a transition temperature from a hypothetic (bcc, gyroid, ...) to the hexagonal phase. The factor in front of $\cos 6 \phi$ in the equation for $\tau_{s}^{(3)}$ is negative. This means that the spinodal temperature is higher for the orientation with $\phi=\pi / 6$. Moreover, the free-energy is minimal for this orientation 
for all values of temperature from the range $\left(\tau_{s}, \tau_{t r}\right)$. Thus we predict the perpendicular orientation to be the only stable orientation in the high-shear limit.

We emphasize that the appearance of the angle-dependence in the free energy only in the $O\left(\beta^{3} \mathfrak{D}^{3}\right)$ is not a coincidence. It reflects the internal symmetry of the system. Any figure on plane can be oriented with respect to the particular direction only if its shape deviates from circle. In other words, if one expands the figure's shape into the plane waves around circle: $r(\phi)=R_{0}\left[1+\sum \alpha_{n} e^{i n \phi}\right]$, the interaction with a selected direction will appear in the first non-zero order. For hexagons it gives $n=3$. The $n$-th order in the expansion corresponds to the interaction between $n$ different wavevectors. Taking into account that the parameter $\beta$ introduces the pare-wise interaction between vectors (see (2.10)), we conclude that the angle-dependent terms in the free energy should be at least $O\left(\beta^{3}\right)$. The role of the parameter $\mathfrak{D}$ is different. Shear breaks the rotational symmetry in the system, stipulating the preferred (gradient) direction and allowing for a discrimination between orientations. Thus the angle-dependent terms in the free energy should be proportional at least to the lowest possible power of $\mathfrak{D}$. This lowest possible power is determined by the concrete form of the structure factor $S(\mathbf{k})$. In (3.2) $\beta$ and $\mathfrak{D}$ enter as a one combination. So, one would expect the free energy to be angle-dependent starting from the $O\left(\beta^{3} \mathfrak{D}^{3}\right)$ order.

We illustrate the concept with the following example in which we calculate the free energy of systems with other rotational symmetry. We consider for the moment an artificial phase which the average density is given by:

$$
C(\mathbf{k})=\sum_{i=1}^{2} a_{i}\left(\delta_{\mathbf{k}, q_{0} \mathbf{n}^{(i)}}+\delta_{\mathbf{k},-q_{0} \mathbf{n}^{(i)}}\right)
$$

where $\mathbf{n}_{i}$ are two vectors lying in the plane perpendicular to the velocity direction, $\mathbf{n}^{(1)} \cdot \mathbf{n}^{(2)}=$ $\cos \theta$. We consider two cases: a) $\theta=\pi / 2$, which describes square packed cylinders and b) $\theta=\pi / 12$, which describes highly non-symmetric cylindrical pattern. Calculating the structure constants (2.12) and integrating (2.17) with the help of (3.2), we obtain for the free energies: 


$$
\begin{aligned}
\Phi_{\theta=\pi / 2}=-\frac{2 \tau^{2}}{3 \lambda}-\frac{4 I_{1} \tau \alpha^{2 / 3}}{3 \lambda^{1 / 3}} \mathfrak{D}-\left[2 I_{1}-\frac{3}{4}\left(I_{3}-I_{2}\right)^{2} \beta^{2} \cos 4 \phi\right] \frac{\alpha^{4 / 3} \lambda^{1 / 3}}{3} \mathfrak{D}^{2} \\
\Phi_{\theta=\pi / 12}=-\frac{2 \tau^{2}}{3 \lambda}+\frac{\tau \alpha^{2 / 3}}{3 \lambda^{1 / 3}} \mathfrak{D}\left\{-4 I_{1}+\right. \\
\left.\frac{\beta}{6}\left[-4(4+\sqrt{3}) I_{1}+12\left(I_{2}+I_{3}\right)+3\left(I_{2}-I_{3}\right)((2+\sqrt{3}) \cos 2 \phi-\sin 2 \phi)\right]\right\}
\end{aligned}
$$

In the case a) the structure deviates from a circle in the second order $\left(\alpha_{0}=\alpha_{1}=0, \alpha_{2} \neq 0\right)$, while in the case b) already in the first order. This gives $\beta^{2}$ and $\beta$ standing in (3.10) and (3.11) in front of the angle-dependent terms. Both cases have the same power of $\mathfrak{D}$ as $\beta$ because of (3.2). In the next section we shall see how a different expression for the fluctuation integral will produce the different lowest possible power of $D$.

\section{WEAK-SHEAR BEHAVIOUR}

In this section we consider the other limit $D \rightarrow 0$. In this case the solution of (2.16)(2.17) only slightly deviates from the equilibrium one and we can construct a perturbation theory with a small parameter $D$. Thus the structure factor is given by the equation:

$$
S(\mathbf{p})=\sum_{n=0}^{\infty}\left(\frac{D}{2 \mu}\right)^{n} S^{(n)}(\mathbf{p}), S^{(n)}(\mathbf{p})=\left[p_{x} S_{0}(\mathbf{p}) \frac{\partial}{\partial p_{y}}\right]^{n} S_{0}(\mathbf{p})
$$

where $S_{0}(\mathbf{p})$ is the equilibrium structure factor.

Following the conclusions of the previous section we evaluate $S(\mathbf{p})$ up to $O\left(\beta^{3}\right)$ and the lowest possible order in $D$, which is $O(D)$. However, $S^{(1)}(\mathbf{p})$ does not contribute to the fluctuation integral. Therefore, we keep $S^{(2)}(\mathbf{p})$ in the expression for the structure factor. Performing integration in (2.13) with $S(\mathbf{p})$ given by (4.1) and using (2.15) for $S_{0}(\mathbf{p})$ we obtain the fluctuation integral:

$$
\sigma(\hat{\mathbf{p}})=\sigma_{e q}(\hat{\mathbf{p}})-\frac{\pi(\alpha \lambda)^{3}}{24 r^{7 / 2}}\left(\frac{D}{D^{*}}\right)^{2}\left\{1+b_{1}+b_{2}+\cdots\right\}, b_{i} \propto O\left(\beta^{i}\right)
$$

where 


$$
\begin{gathered}
b_{1}=\frac{1}{2 r}\left(3 e_{x x}+3 e_{y y}+e_{z z}\right)+\beta \frac{2 \hat{p}_{z}^{2}-3}{7} \\
b_{2}=\frac{3}{8 r^{2}}\left[2 e_{i j} e_{j i}+e_{i i}\left(3 e_{x x}+3 e_{y y}-e_{z z}\right)\right]- \\
\frac{\beta}{3 r}\left[\hat{p}_{i} e_{i j} \hat{p}_{j}+\frac{1}{2} e_{i i}+\left(1-\hat{p}_{z}^{2}\right)\left(e_{x x}+e_{y y}\right)-e_{z z} \hat{p}_{z}^{2}\right] \\
\sigma_{e q}(\hat{\mathbf{p}})=\frac{\alpha \lambda}{\sqrt{r}}\left[1-\frac{1}{3} \beta+\frac{e_{i i}}{6 r}\right]+\frac{\alpha \lambda}{10 r^{3 / 2}}\left[\frac{1}{4 r}\left(e_{i i}^{2}+2 e_{i j} e_{j i}\right)-\frac{\beta}{3}\left(e_{i i}+2 \hat{p}_{i} e_{i j} \hat{p}_{j}\right)\right]+\cdots
\end{gathered}
$$

and $\sigma_{e q}$ is an extension of (2.19) to the higher orders in $\beta$. Here we assumed the summation over repeated indices.

In the equation (4.2) and in what follows the terms of $O\left(\beta^{3}\right)$ are cumbersome and we do not present them here. However, we did use them in our calculations.

The $\mathbf{p}$-independent terms in (4.2) contribute to the equation for $r$ :

$$
r=\tau+\lambda A+\frac{\alpha \lambda}{\sqrt{r}} R_{1}-\left(\frac{D}{D^{*}}\right)^{2} \frac{(\alpha \lambda)^{3} \pi}{24 r^{7 / 2}} R_{2}
$$

where

$$
\begin{gathered}
R_{1}=1+\frac{e_{i i}}{6 r}+\frac{1}{40 r^{2}}\left(e_{i i}^{2}+2 e_{i j} e_{j i}\right)+\cdots \\
R_{2}=1+\frac{3 e_{x x}+3 e_{y y}+e_{z z}}{2 r}+3 \frac{e_{i i}\left(3 e_{x x}+3 e_{y y}-e_{z z}\right)+2 e_{i j} e_{j i}}{8 r^{2}}+\cdots
\end{gathered}
$$

For the anisotropy tensor (4.2) together with (2.14) gives a closed set of equations. Iterating them up to $O\left(D^{2}\right)$ and $O\left(\beta^{3}\right)$ we obtain:

$$
e_{i j}=E_{i j}^{(1)}-\left(\frac{D}{D^{*}}\right)^{2} \frac{(\alpha \lambda)^{3} \pi}{24 r^{7 / 2}} E_{i j}^{(2)}+\cdots
$$

where

$$
\begin{aligned}
E_{i j}^{(1)}= & \beta\left\{\frac{\alpha \lambda}{3 \sqrt{r}} \delta_{i j}+\lambda \sum_{s=1}^{3} a_{s}^{2} n_{i}^{(s)} n_{j}^{(s)}\right\}+ \\
& \beta^{2}\left\{\frac{(\alpha \lambda)^{2}}{18 r^{2}} \delta_{i j}+\frac{\alpha \lambda^{2}}{30 r^{3 / 2}} \sum_{s=1}^{3} a_{s}^{2}\left[\delta_{i j}+2 n_{i}^{(s)} n_{j}^{(s)}\left(2-\delta_{i j}\right)\right]\right\}+\cdots \\
E_{i j}^{(2)}= & \frac{\beta}{7}\left\{3 \delta_{i x} \delta_{j x}+3 \delta_{i y} \delta_{j y}+\delta_{i z} \delta_{j z}\right\}+\beta^{2}\left\{\frac{\alpha \lambda}{105 r^{3 / 2}}\left[59\left(\delta_{i x} \delta_{j x}+\delta_{i y} \delta_{j y}\right)+22 \delta_{i z} \delta_{j z}\right]+\right. \\
& \left.\frac{\lambda}{6 r} \sum_{s=1}^{3} a_{s}^{2}\left[\delta_{i j}+2 n_{y}^{(s) 2}\left(\delta_{i x} \delta_{j x}+2 \delta_{i y} \delta_{j y}\right)+4 n_{i}^{(s)} n_{j}^{(s)}\left(1-\delta_{i j}\right)\right]\right\}+\cdots
\end{aligned}
$$


Equations (4.3) and (4.4) are enough to construct the free energy $\Phi$ as a solution of (2.22), where $h_{i}, r$ and $e_{i j}$ are given by (2.17), (4.3) and (4.4), respectively. Integration of (2.22) using (2.23) leads to the following equation for the free energy:

$$
\Phi=F-\beta^{3}\left(\frac{D}{D^{*}}\right)^{2} \mathfrak{a}^{4} \frac{3 \pi \alpha^{4} \lambda^{5}}{1280 r_{0}^{6}} \cos 6 \phi
$$

where $\mathfrak{a}$ is the equilibrium amplitude given by the equations (2.29) and (2.27). In (4.5) $F$ stands for the angle-independent part of the free energy.

The spinodal temperature $\tau_{s}$ is given by:

$$
\tau_{s}=t+\beta^{3}\left(\frac{D}{D^{*}}\right)^{2} \mathfrak{a}^{4} \frac{\pi \alpha^{4} \lambda^{6}}{640 r_{0}^{7}} \cos 6 \phi
$$

where $t$ includes the angle-independent terms. The spinodal temperature is maximal for $\phi=0$. At the same time, the free energy is minimal for the same orientation. It means that only the parallel orientation is stable under low shear.

To finish our symmetry analysis we note that in principle the fluctuation integral (4.2) contains all possible combinations of powers of $\beta$ and $D^{2}$. According to the symmetry arguments the angle-dependence appears in the lowest possible order in $D$ (which is $D^{2}$ in this case) and the third order in $\beta$. This is in agreement with (4.5) and (4.6).

\section{CONCLUSIONS}

In the present paper we have shown how the orientational behaviour of the hexagonal phase under simple shear flow can be described in the framework of the dynamical model first developed by Fredrickson for the lamellae [1]. In this model the angle-dependence of the 4th order vertex function $\lambda$ (see 2.10) plays a crucial role, although it is of no importance in equilibrium (see Section II for details). The parameter $\beta$ introduces the interaction between structure and shear flow and allows for the rotational symmetry breaking. The character of the interaction depends on the shear rate and the resulting phase diagram has a complex structure. We predict the parallel orientation to be stable at low and the perpendicular orientation at high shear rates. Our results are in agreement with the experimental dynamical 
phase diagram (Fig.2) for the PE-PEP system [7]. Our analysis shows that experimental study of the hexagonal phase orientation is actually very difficult to perform. Because of the symmetry arguments, the difference in the free energies of the different orientations $\Delta \Phi=\Phi_{\perp}-\Phi_{\|}$is proportional to

$$
\Delta \Phi=\left\{\begin{array}{l}
\beta^{3} \frac{D^{*}}{D}, D \rightarrow \infty \\
\beta^{3}\left(\frac{D}{D^{*}}\right)^{2}, D \rightarrow 0
\end{array}\right.
$$

This difference is extremely small (compare with $\Delta \Phi \propto \beta$ for lamellae [1]) and a particular orientation is only slightly more stable than the other one. Some crude experimental by-effects (like the ordering influence of the sample walls) can easily suppress the pure orientational behaviour we discussed.

In comparison with the work [1] we ignored the hydrodynamic corrections to the effective shear rate $D_{\text {eff }}$ (see Eq.(2.27) in [四). These corrections are responsible for the $\perp \rightarrow \|$ transition in the lamellar phase under strong shear. Taken into account they would probably lead to the same transition in the hexagonal phase. However, this is not an easy task because one should keep terms up to $O\left(\beta^{3} \mathfrak{D}^{3}\right)$ in order to have an angle-dependent value.

Another remaining problem concerns the stability of transversal cylinders, i.e. cylinders with their axis oriented not in the direction of flow [4]. Our model cannot describe stability of the transversal orientation [3]. Another approach should be developed to complete our study.

\section{ACKNOWLEDGMENTS}

We gratefully acknowledge Glenn Fredrickson for explaining the details of his work [1]. The luminous discussions with A. V. Zatovsky and V. M. Adamjan helped us to understand the role of hydrodynamics and develop the theory group approach presented in Sections II and III. We also want to thank D. Bedeaux and V. L. Koulinskii for the general remarks on the subject, and Agur Sevink for the friendly atmosphere in our group. 
Our special thanks are to Nicolay Malomuzh for his enormous influence and kind advice. 


\section{REFERENCES}

[1] G. H. Fredrickson, J. Rheol. 38, 1045 (1994).

[2] K. A. Koppi, M. Tirrell, F. S. Bates, K. Almdal and R. H. Colby, J. Phys France 2, 1941 (1992).

[3] M. E. Cates and S. T. Milner, Phys. Rev. Lett. 62, 1856 (1989).

[4] G. H. Fredrickson and F. S. Bates, Ann. Rev. Mater. Sci. 26, 501 (1996).

[5] L. Leibler, Macromolucules 13, 1602 (1980).

[6] G. H. Fredrickson and E. Helfand, J. Chem. Phys. 87, 697 (1987).

[7] T. Tepe, M. F. Schulz, J. Zhao, M. Tirrell and F. S. Bates, Macromolecules 28, 3008 (1995).

[8] G. Hadziioannou, A. Mathis and A. Skoulios, Colloid \& Polymer Sci. 257, 15 (1979);

G. Hadziioannou, A. Mathis and A. Skoulios, Colloid \& Polymer Sci. 257, 136 (1979).

[9] G. Schmidt, W. Richtering, P. Lindner, and P. Alexandridis, Macromolecules 31, 2293 (1998); W. Richtering, private communication.

[10] A. Onuki and K. Kawasaki, Ann. Phys. 121, 456 (1979).

[11] K. Kawasaki and K. Sekimoto, Macromolecules 22, 3063 (1989).

[12] C. M. Marques and M. E. Cates, J. Phys. France 51, 1733 (1990).

[13] L. D. Landau and E. M. Lifshitz, Statistical Physics: part I (Pergamon, New York, 1980).

[14] The only role of the dissipation in our model is to renormalize the shear rate $D \rightarrow$

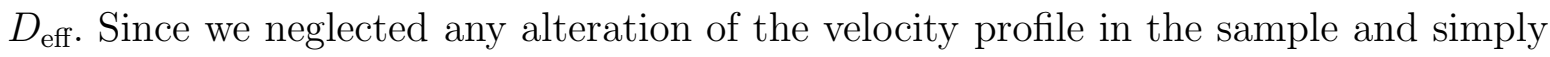
approximated $D_{\text {eff }} \approx D$, the existence of the potential $\Phi$ is not surprising. Moreover, $D_{\text {eff }}$ is a functional of the dissipative effects [1], so that only the number $D_{\text {eff }}$ enters the 
dynamic equations. Thus, even in this case the equations for the amplitudes $a_{i}$ have a potential form. However, for the dissipative case it is much more difficult to speculate about physical meaning of $\Phi$.

[15] S. A. Brazovskii, I. E. Dzyaloshinskii and A. R. Muratov, Sov. Phys. JETP 66, 625 (1987).

[16] E. I. Kats, V. V. Lebedev and A. R. Muratov, Phys. Rep. 228, 1 (1993).

[17] D. C. Morse and S. T. Milner, Phys. Rev E 47, 1119 (1993).

[18] S. A. Brazovskii, Sov. Phys. JETP 41, 85 (1975). 


\section{FIGURES}

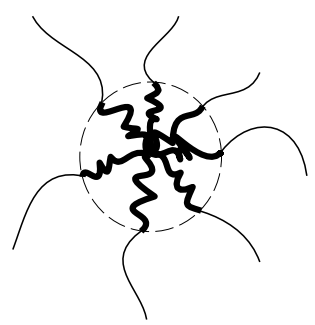

FIG. 1. The molecular structure of a cylinder

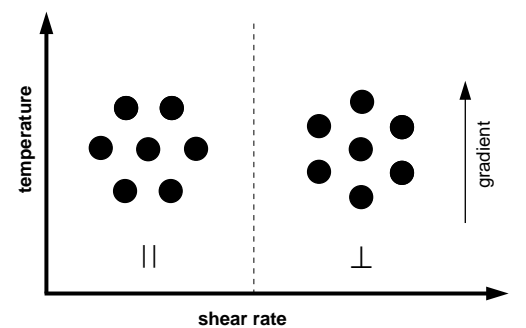

FIG. 2. Schematic dynamical phase diagram of hexagonal phase under shear flow: the parallel $(\phi=0)$ and perpendicular $(\phi=\pi / 6)$ orientations (see eq.2.11)
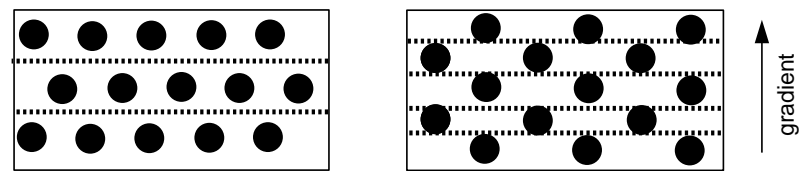

FIG. 3. Conventional subdivision of hexagonal pattern in lamellar layers

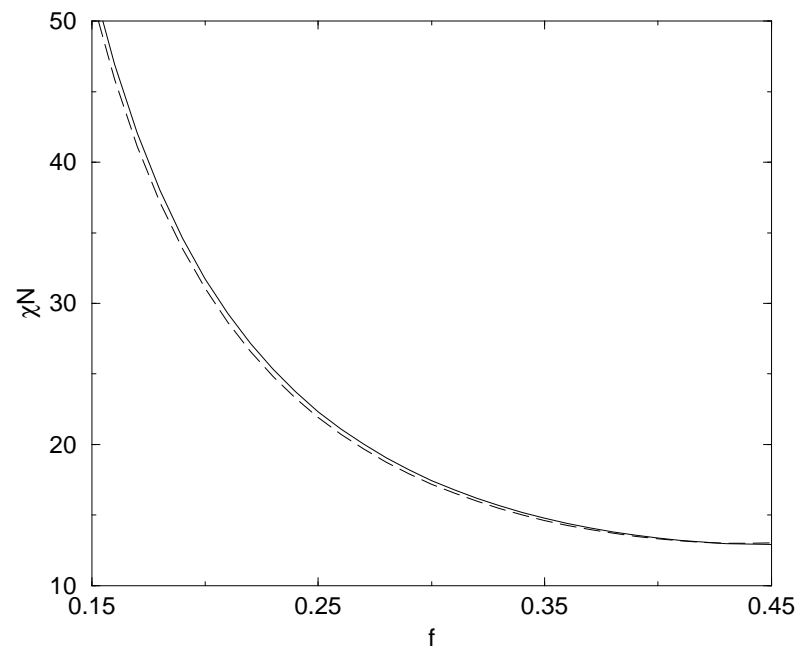


FIG. 4. The spinodal temperature (solid line: $\beta=0$, dashed line: $\beta=0.5$ )

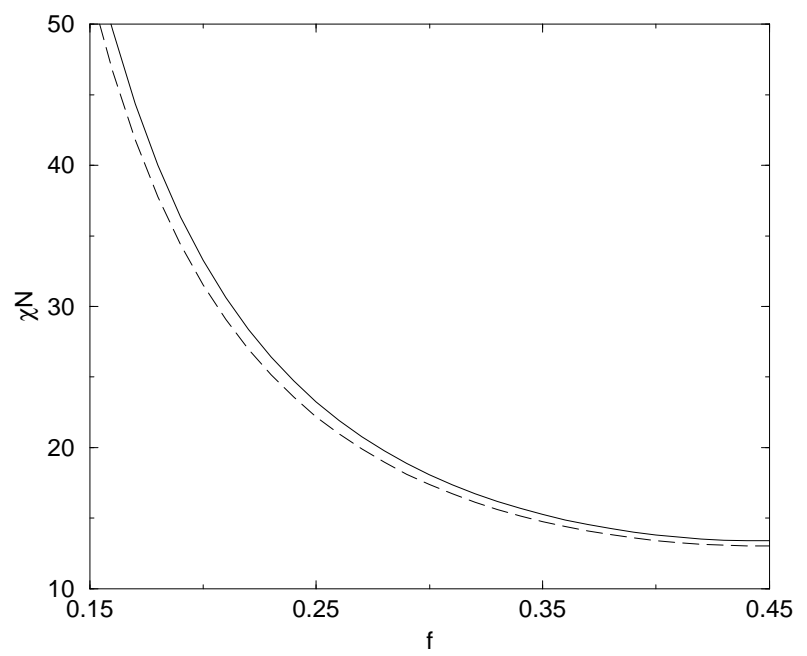

FIG. 5. The transition temperature between lamellae and cylinders (solid line: $\beta=0$, dashed line: $\beta=0.5)$

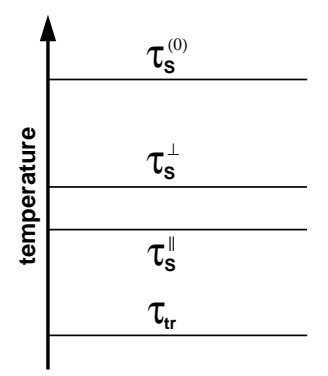

FIG. 6. Region of stability of the hexagonal phase in high shear flow 